\title{
Laboratory Evaluation of Dynamic Routing of Air Traffic in an En Route Arrival Metering Environment
}

Doug Isaacson, Miwa Hayashi, Chester Gong, Huabin Tang, \& Gregory Wong NASA Ames Research Center Moffett Field, CA, USA

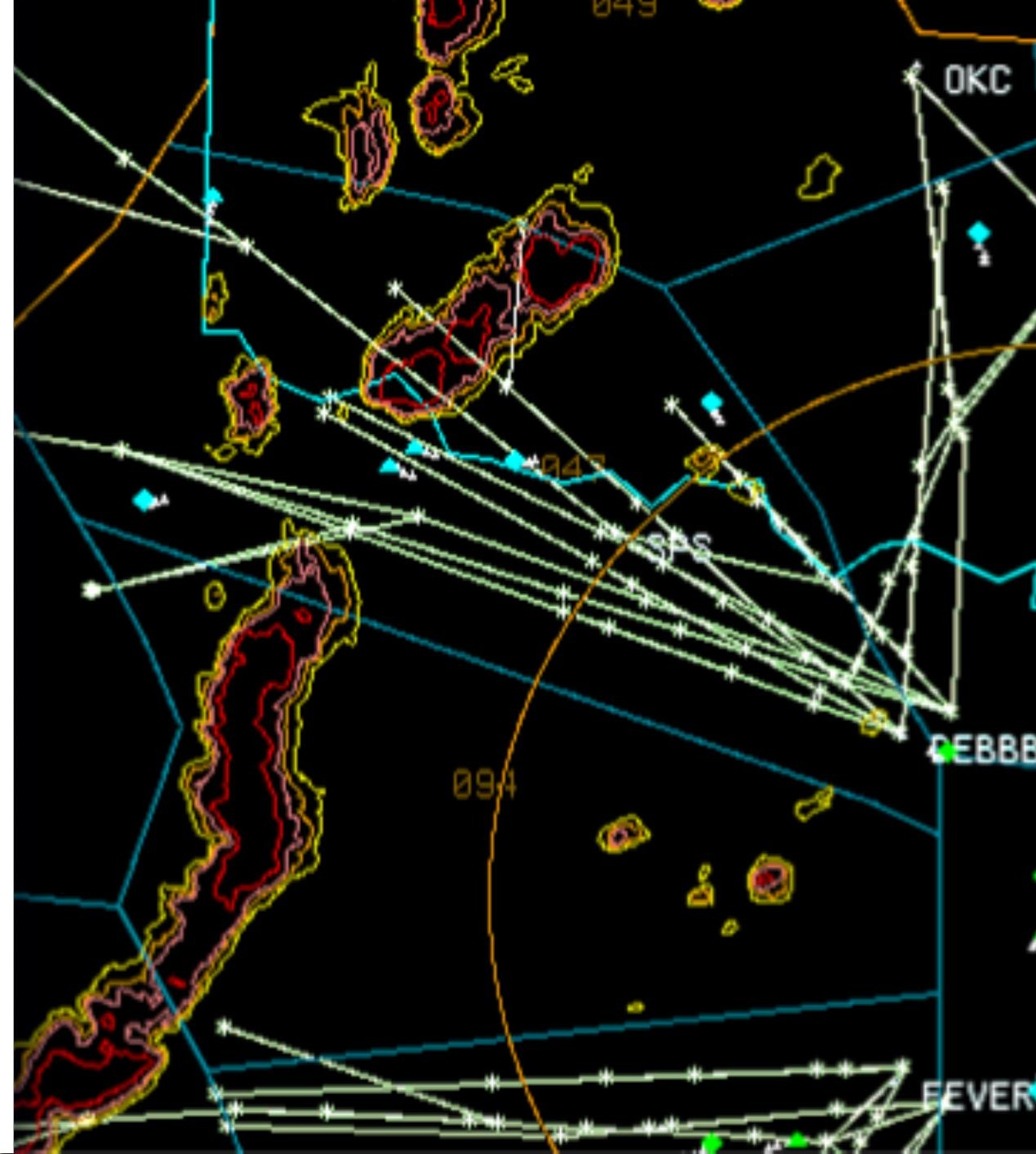




\section{Contents}

1. Background

2. Dynamic Routing for Arrivals in Weather (DRAW)

3. Laboratory Evaluation

4. Results

5. Conclusion 


\section{1. Background}

- Challenges

- Solution

- Past Work 


\section{Challenges}

- In clear weather

- Efficient, precision air traffic flow management.

- However, when convective weather is present...

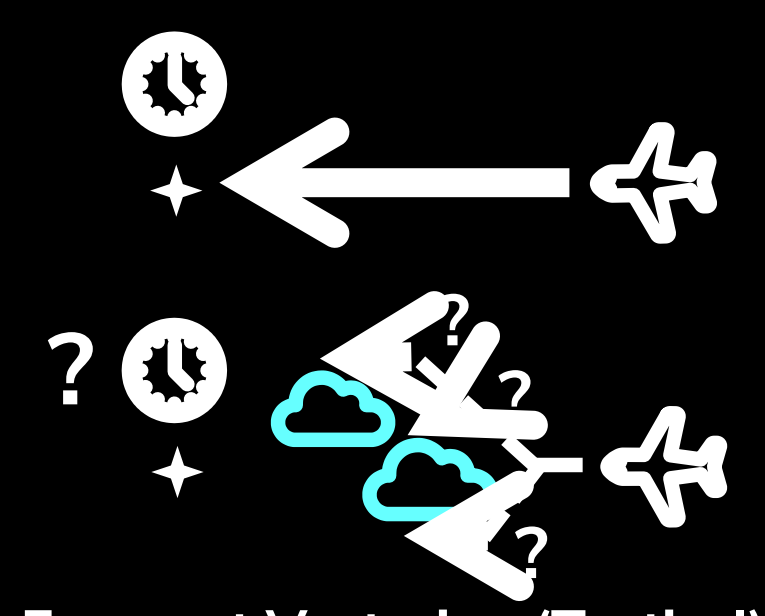

Frequent Vectoring (Tactical)

Less predictable High workload

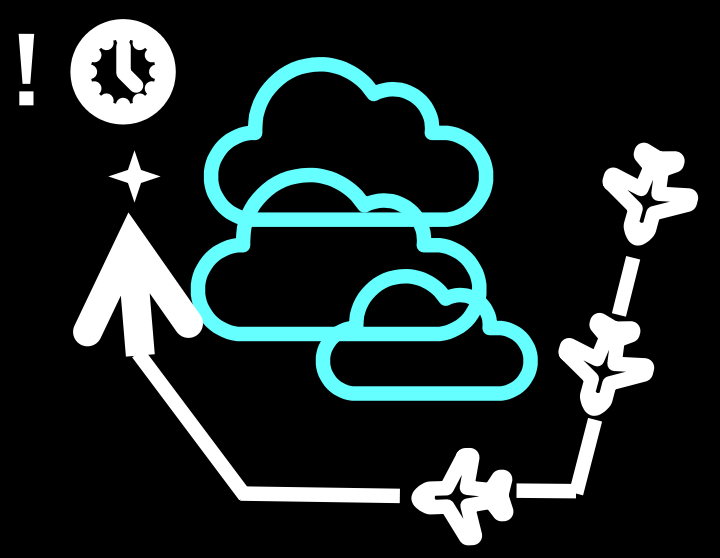

Playbook (Strategic)

Inefficient ("One-size-fits-all")

Slow to respond 
- Desired: Rerouting tool that...

- Avoids weather

- Is more predictable $\&$ responsive

- Solution:

- Dynamic rerouting (Flight Plan amendment) 


\section{Past Work}

Dynamic routing in weather
- MIT Lincoln Lab's Convective Weather Avoidance Model (CWAM) [DeLaura, et. al, 2008]

- MIT Lincoln Lab's Route Availability Planning Tool (RAPT) and the Arrival Route Status and Impact [Robinson, DeLaura, \& Underhill, 2009]

- NASA's Dynamic Weather Routes (DWR) [McNally, et. al, 2015] 
2.

Dynamic Routing for Arrivals in Weather (DRAW)

- Concept

- Components

- Example

- User Process 
DRAW Concept
Dynamic Routing for Arrivals in Weather (DRAW):

- Adapted from DWR

- Designed for Traffic Management Coordinator (TMC) at FAA ARTCC ("Center")

- Reroutes arrivals for weather avoidance

- Supports arrival-metering operations 
DRAW

Components

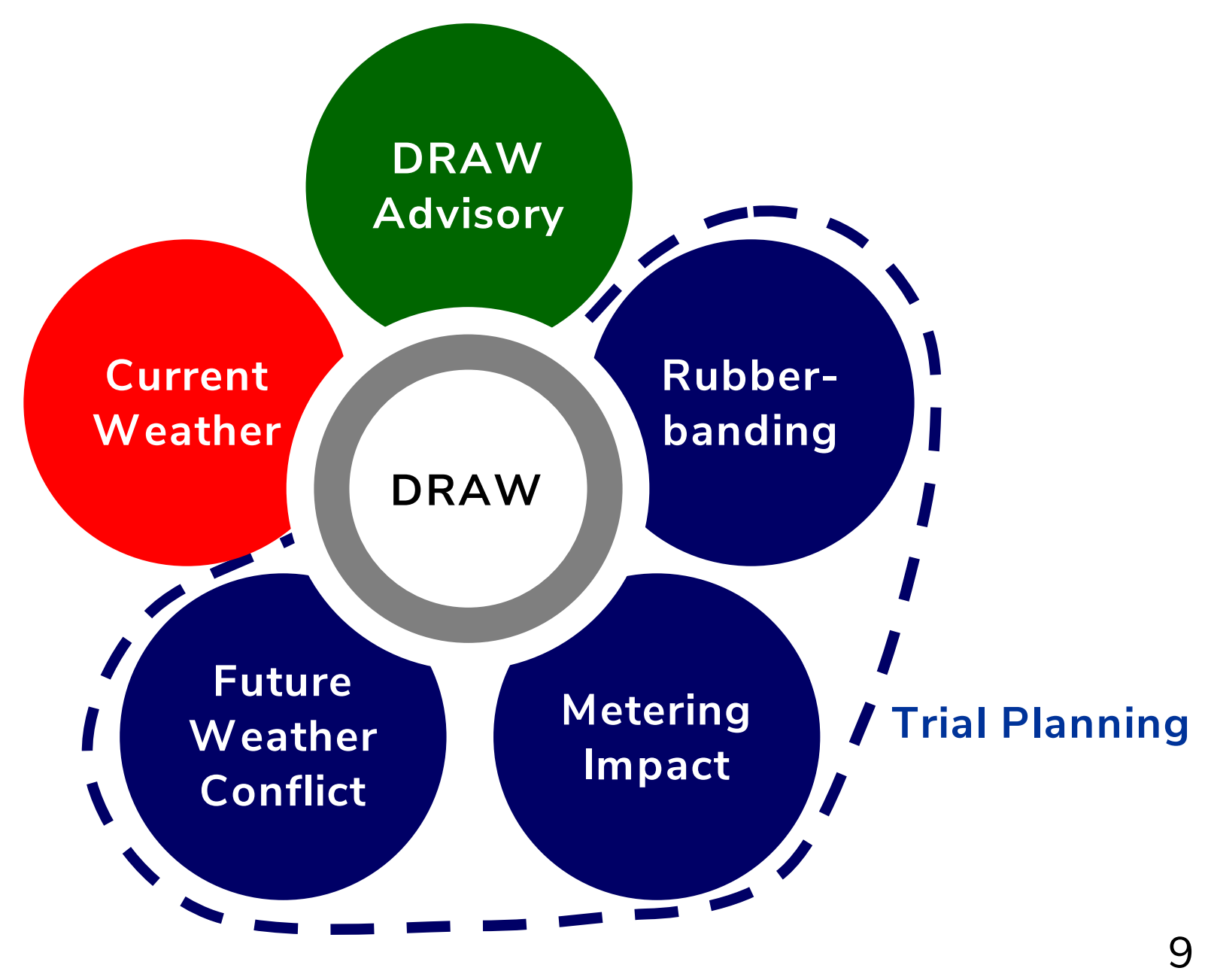




\section{Example of DRAW Advisory and Trial Planning}

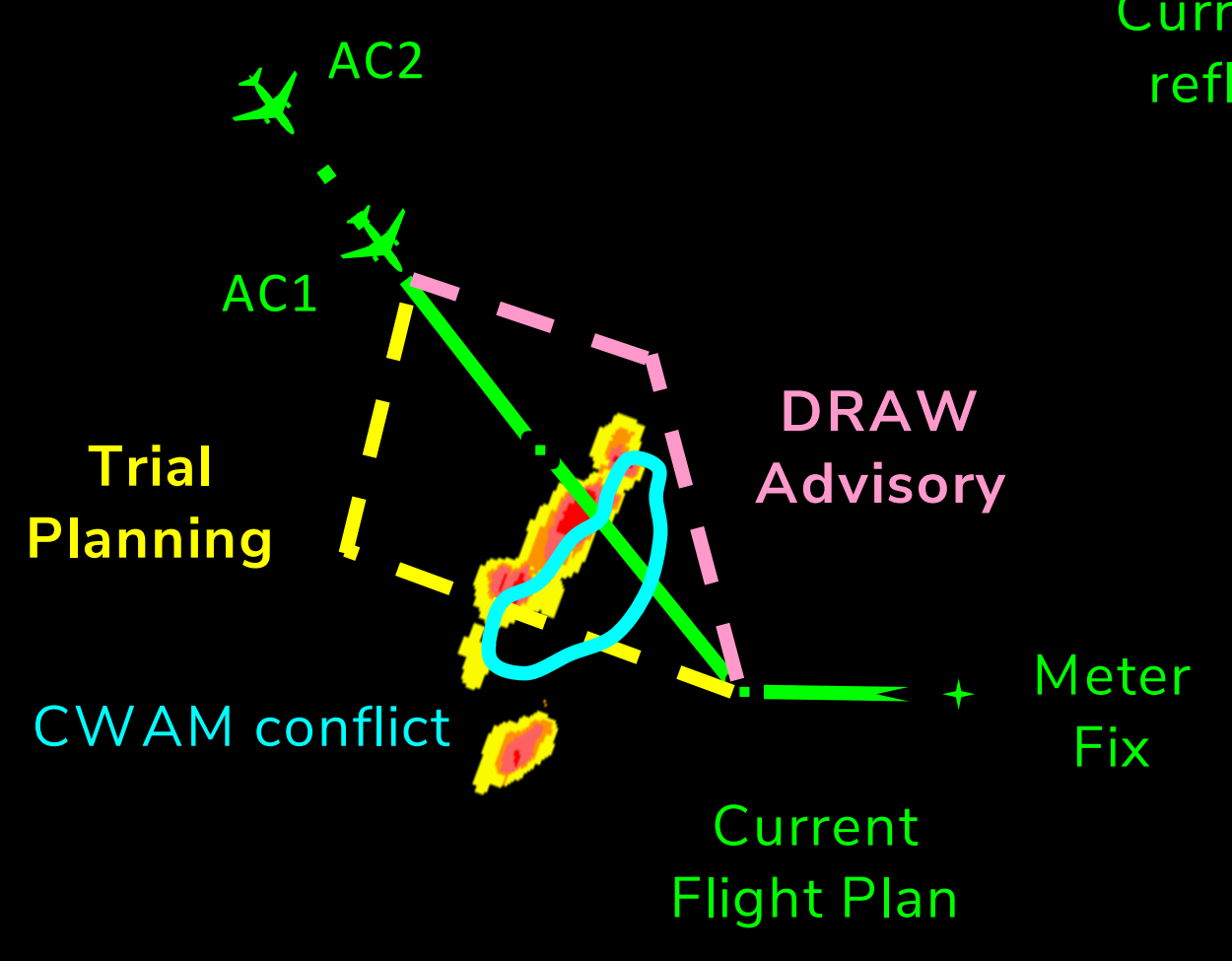

Current scheduled times of arrival do not reflect the need to deviate for weather

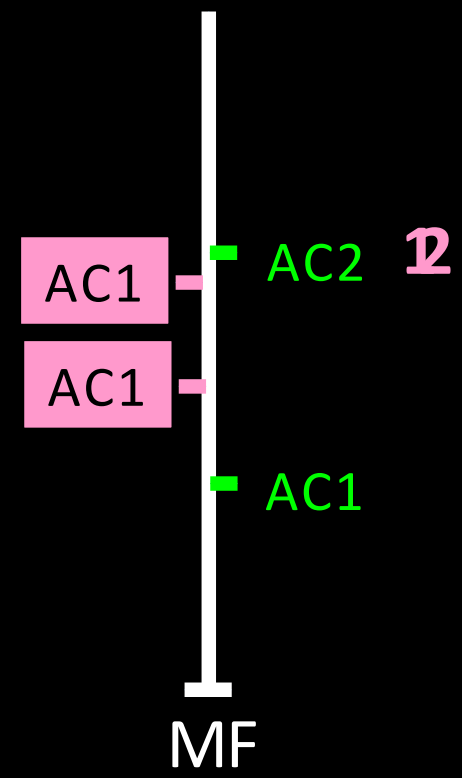




\section{DRAW \\ User Process}

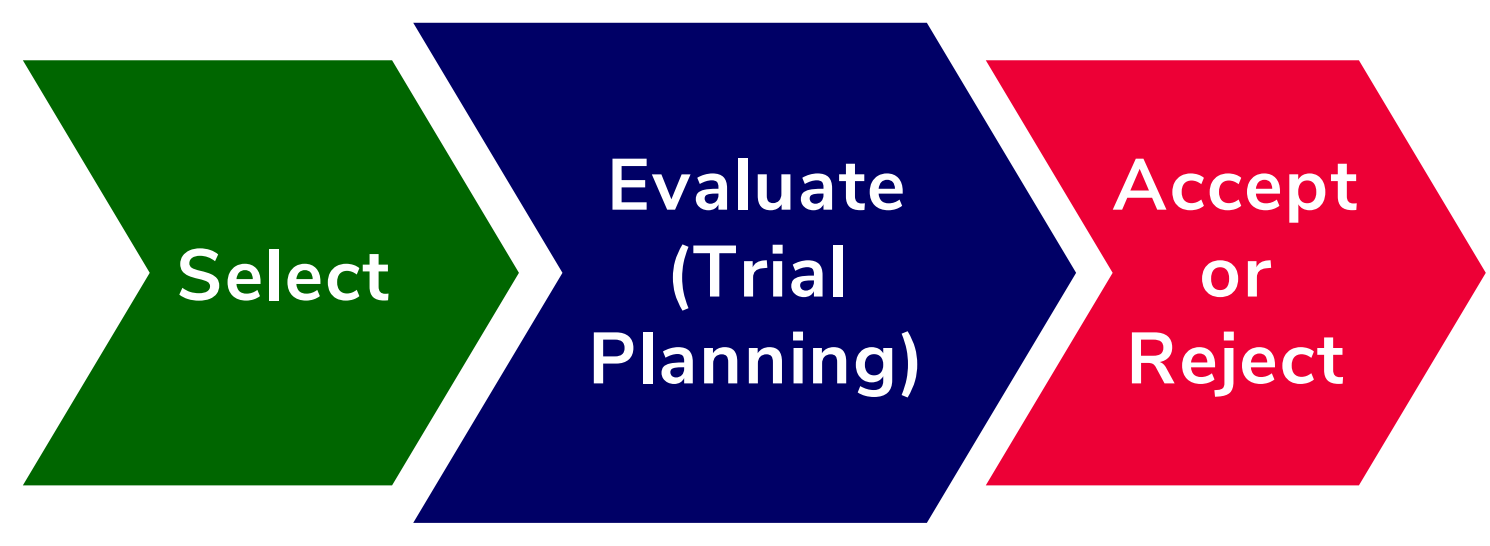

Accept $\rightarrow$ Flight Plan amendment 


\section{3.}

\section{Laboratory}

Evaluation

- Airspace

- Experiment Design

- Lab Setup 


\section{Fort Worth Center (ZFW) Airspace}

Bowie and Glen Rose arrivals to DFW/DAL of ZFW were simulated.

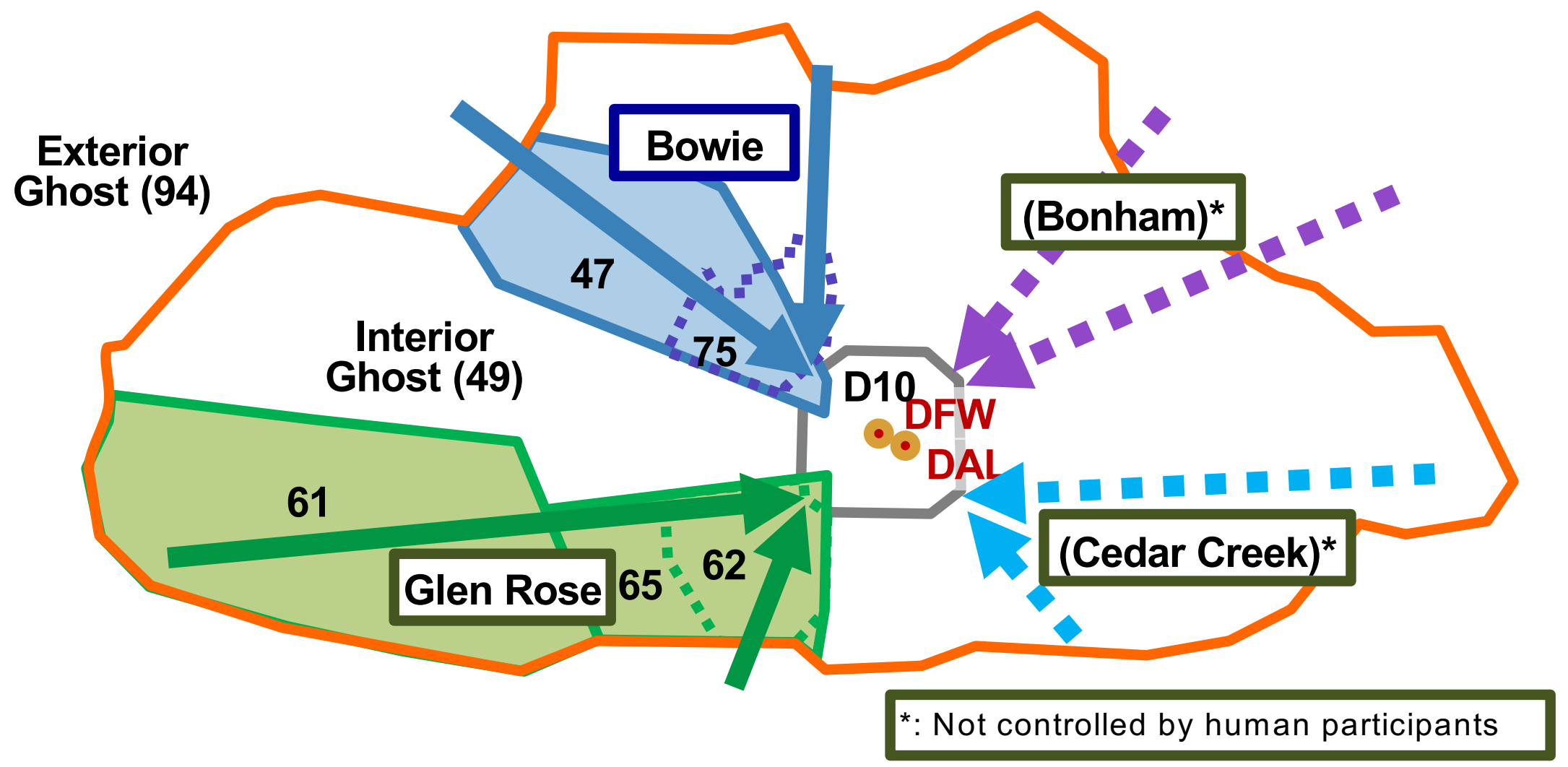


Experiment Design
- TMC Sessions (32 runs) and Controller Session (16 runs) conducted separately

- Independent Variables:

- 2 DRAW conditions (DRAW vs. No-DRAW)

- 2 Weather Scenarios

- 4 TMCs (2 TMCs in Controller Session)

- 2 Controller Seating Positions (Controller Session)

- Clear-weather day traffic

- Assumed: all FP amendments instantly executed 


\section{DRAW vs. No-DRAW Conditions}

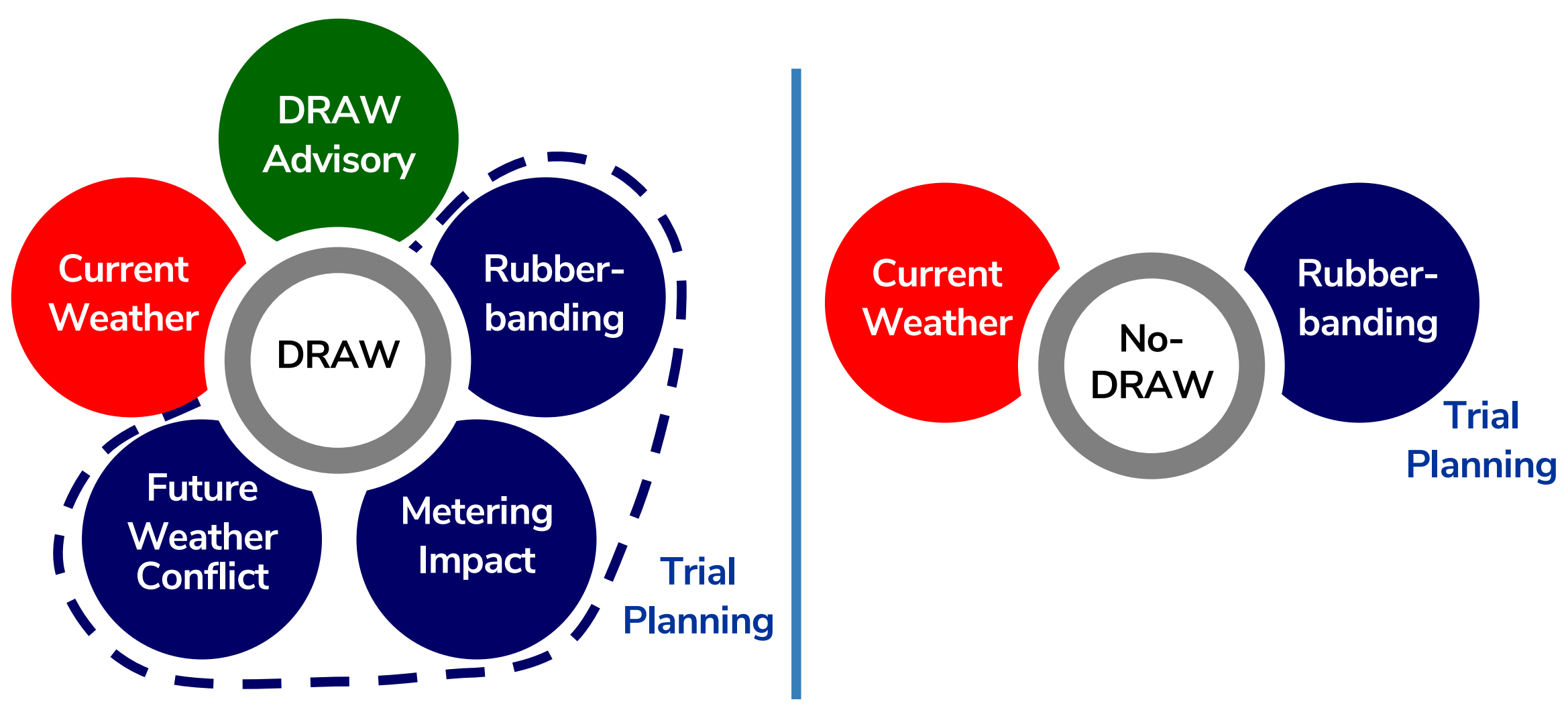




\section{Lab Setup}

TMC Workstations

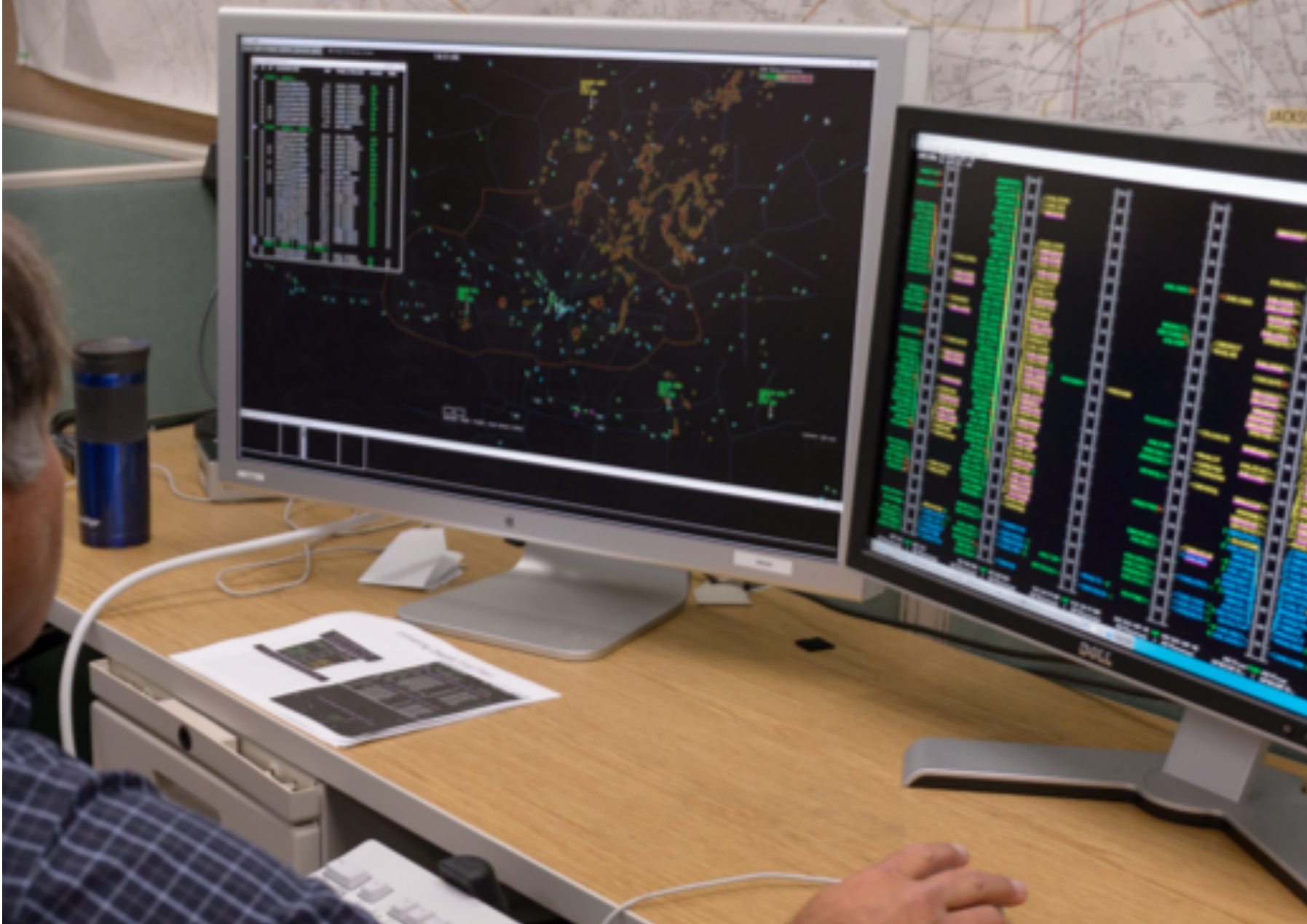




\section{Lab Setup}

Sector Controller

Workstations

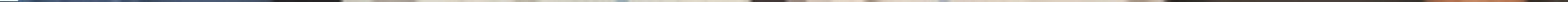




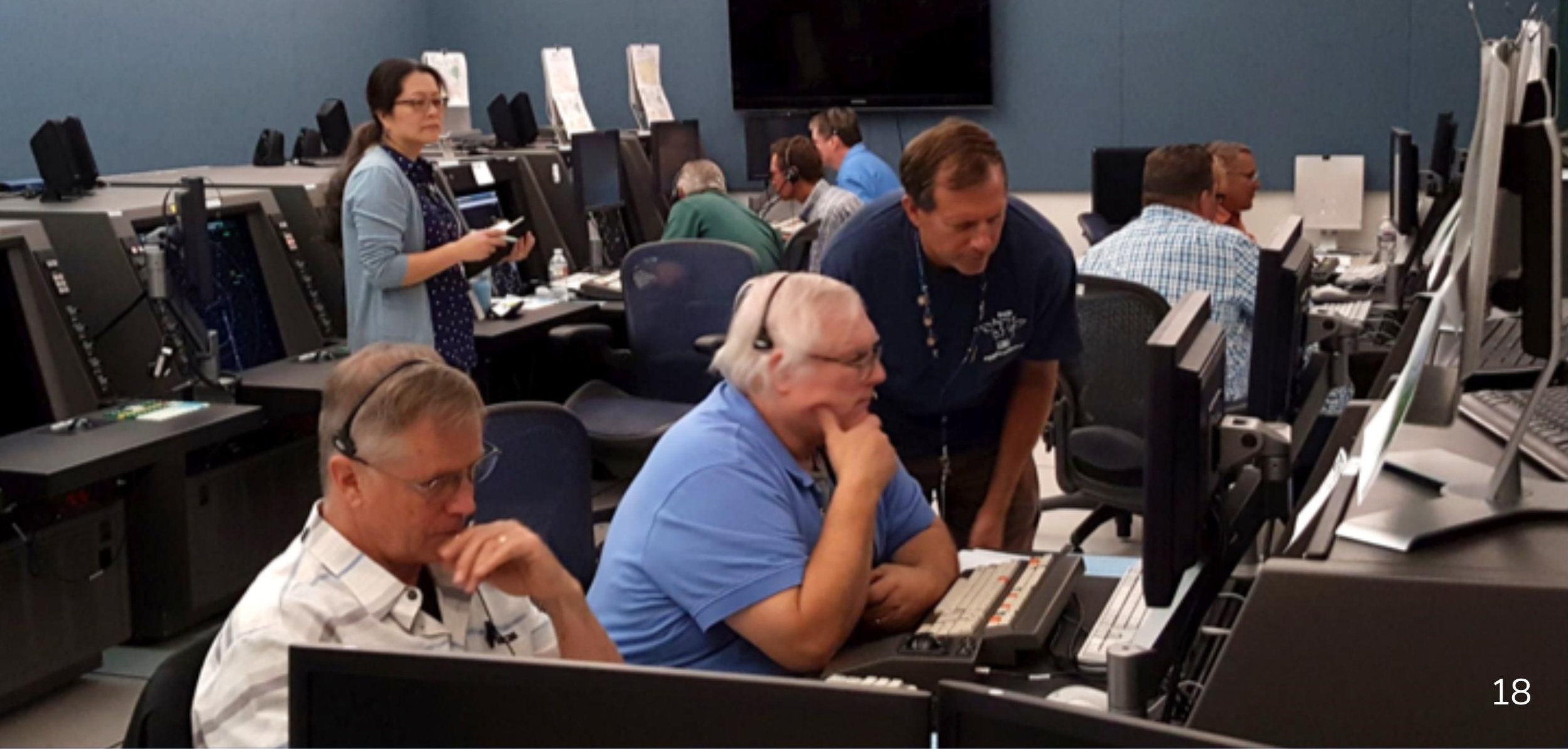


2

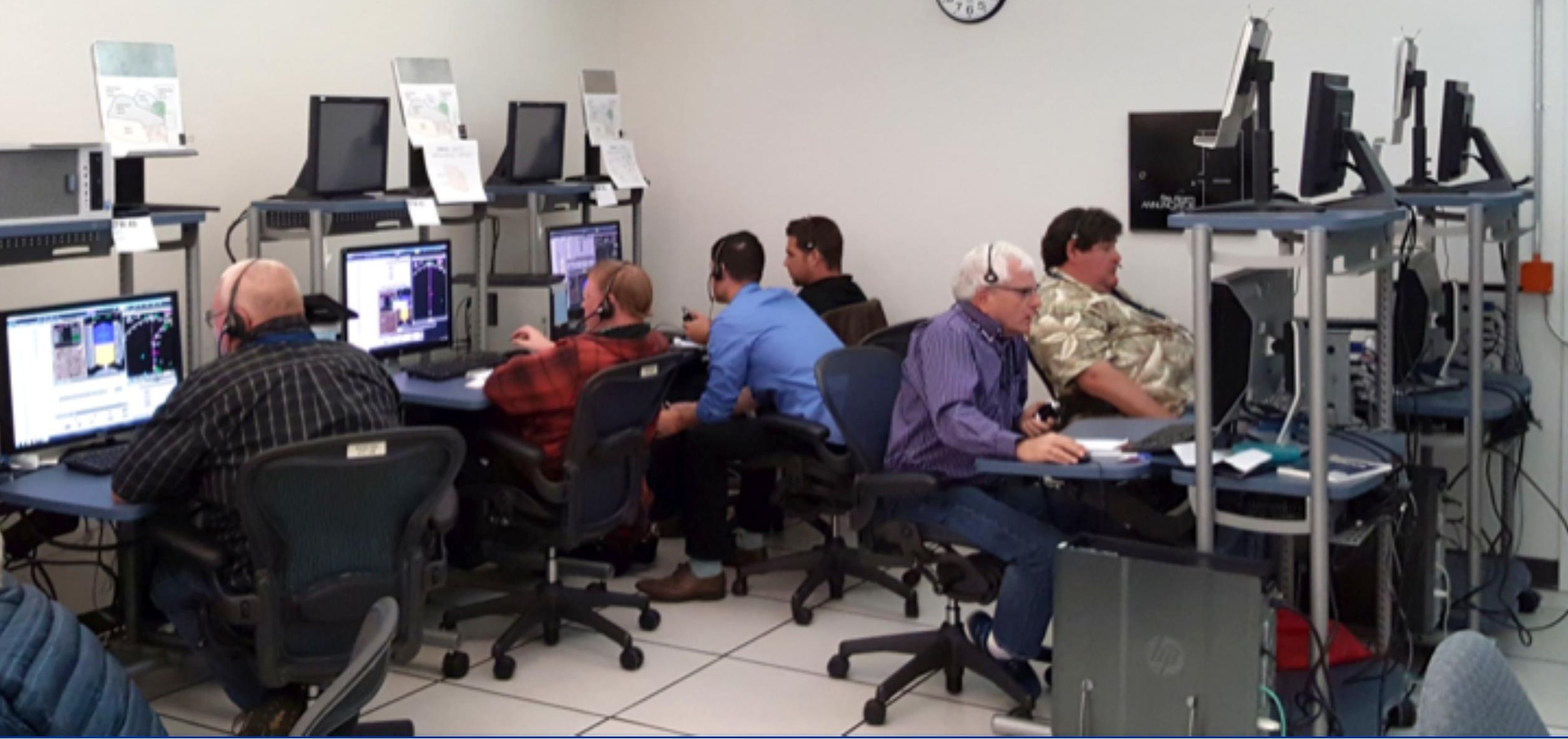




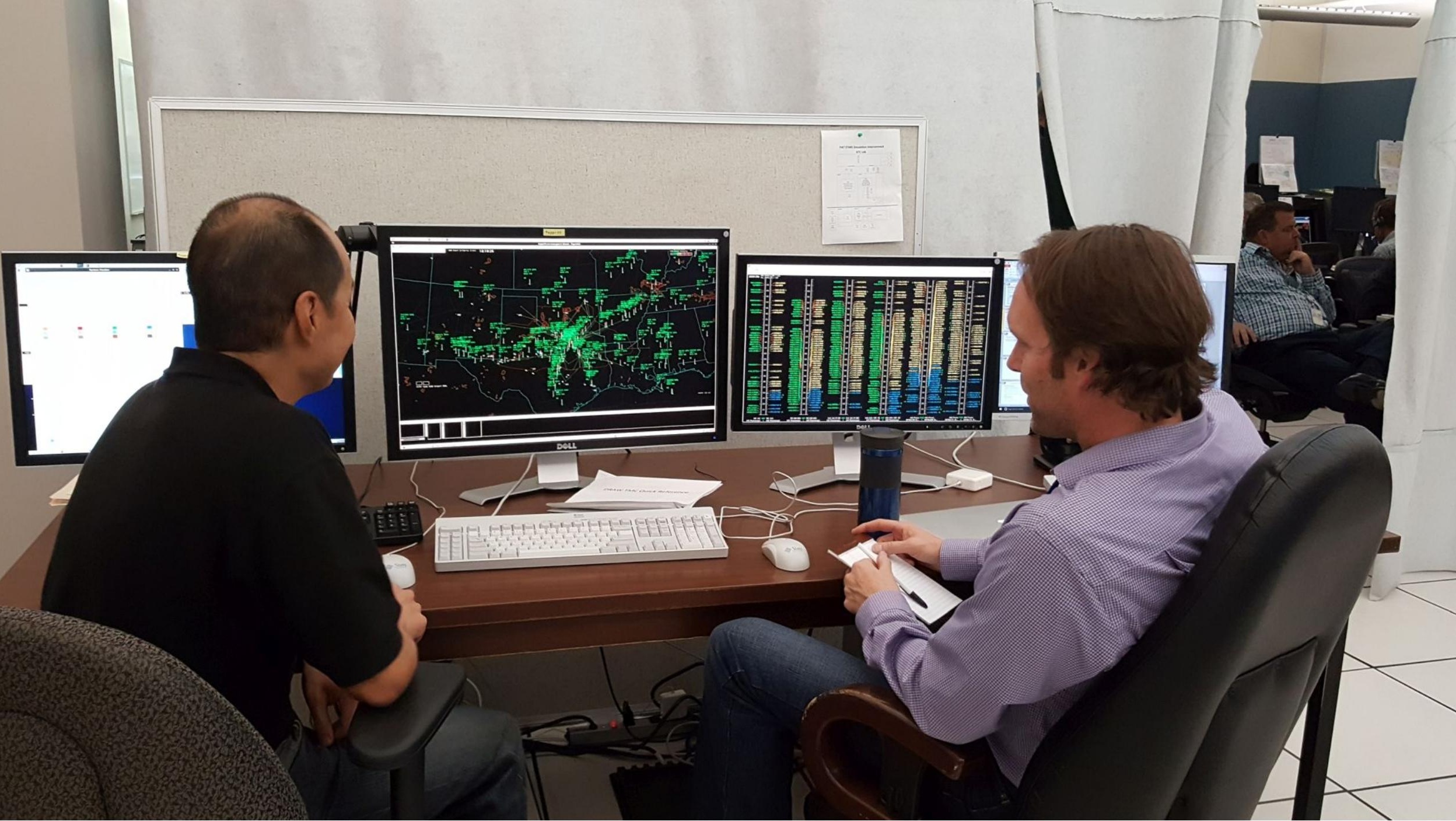


4.

\section{Results}

- Reroute Timing

- Weather Avoidance

- TMC Acceptability

- Controller Workload 
Reroute Timing

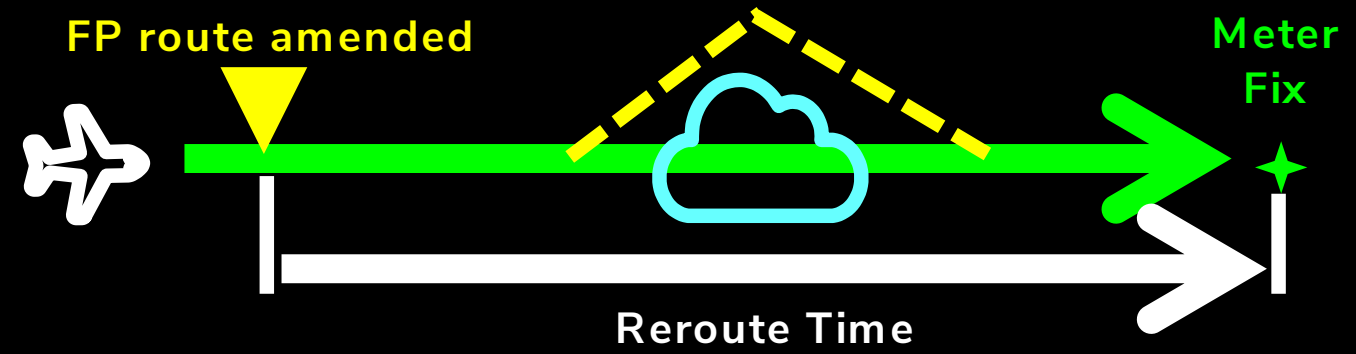

TMCs rerouted earlier when using DRAW $(p=0.001)$.

- Mean $=82 \mathrm{~min}$ in DRAW runs

- Mean $=66 \mathrm{~min}$ in No-DRAW runs 


\section{Weather Avoidance}

DRAW reduced the number of flights that had residual weather conflicts in the Center airspace $(p=0.017)$.

- Mean $=5.6$ flights per DRAW run

- Mean $=10.8$ flights per No-DRAW run

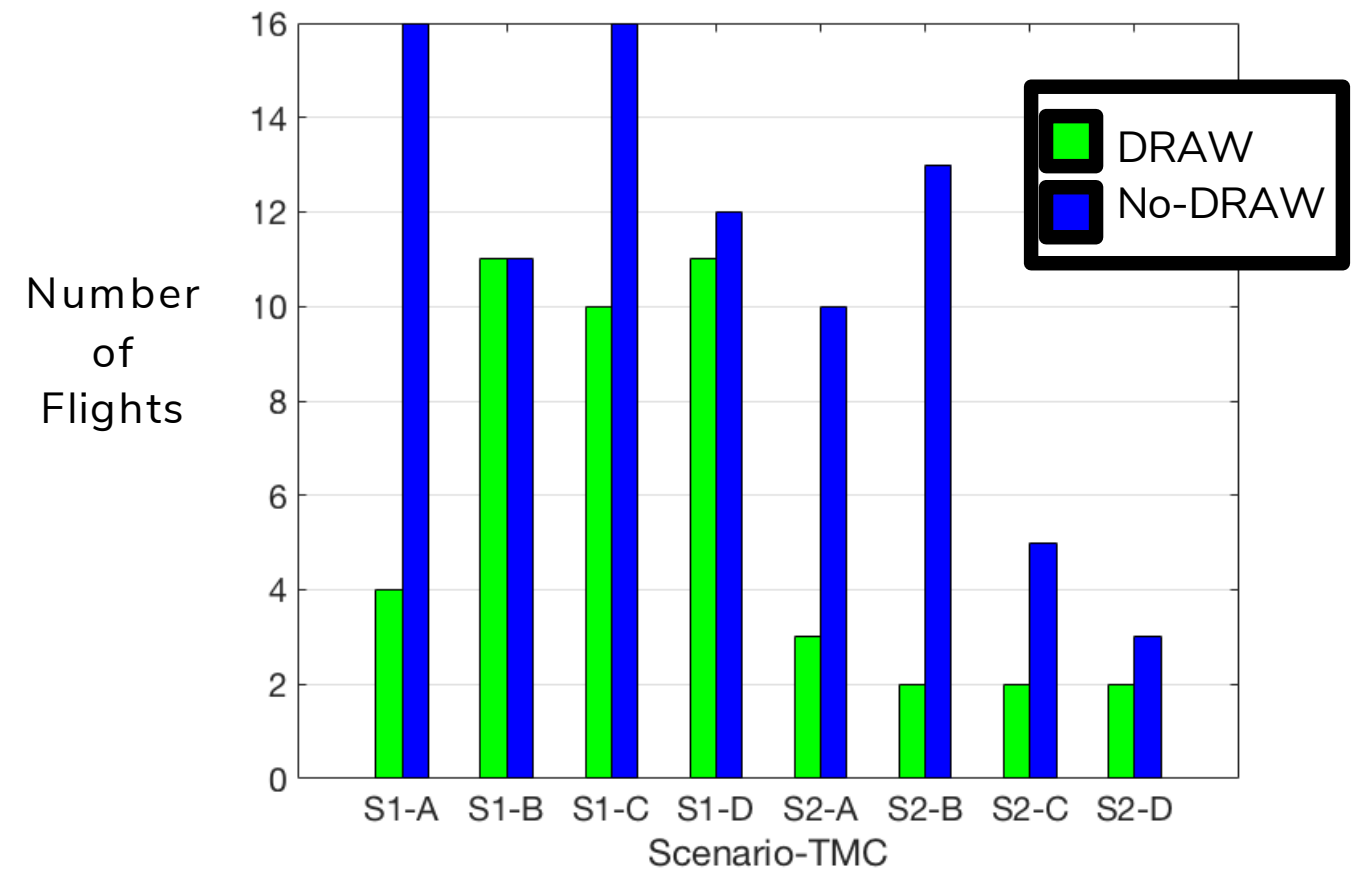




\section{TMC} Acceptability
TMC Post-run questionnaire responses results:

1 = Strongly Disagree, 4 = Neutral, 7 = Strongly Agree

- Mean Rating = 6 7 ("Agree" to "Strongly Agree")

- DRAW workload was acceptable.

- DRAW advisory timing was early enough.

- DRAW was helpful in arrival traffic management in weather.

- Mean Rating = 4 5 ("Neutral" to "Somewhat Agree")

- DRAW would increase probability of sustaining arrival metering in weather.

- DRAW would delay the need for other Traffic Management Initiatives (e.g., Miles-in-Trail, Playbook). 


\section{Controller Workload}

- Controller post-run questionnaire collected their NASA TLX workload ratings:

- Linear Mixed Model regression analysis found that in DRAW runs...

- Sector 47 controller's mental workload demand was reduced ( $p=0.029)$.

- Controllers felt their performance level poorer ( $p=0.048)$.

- No other DRAW effect was found. 
5.

Conclusions

- Summary

- Future Work

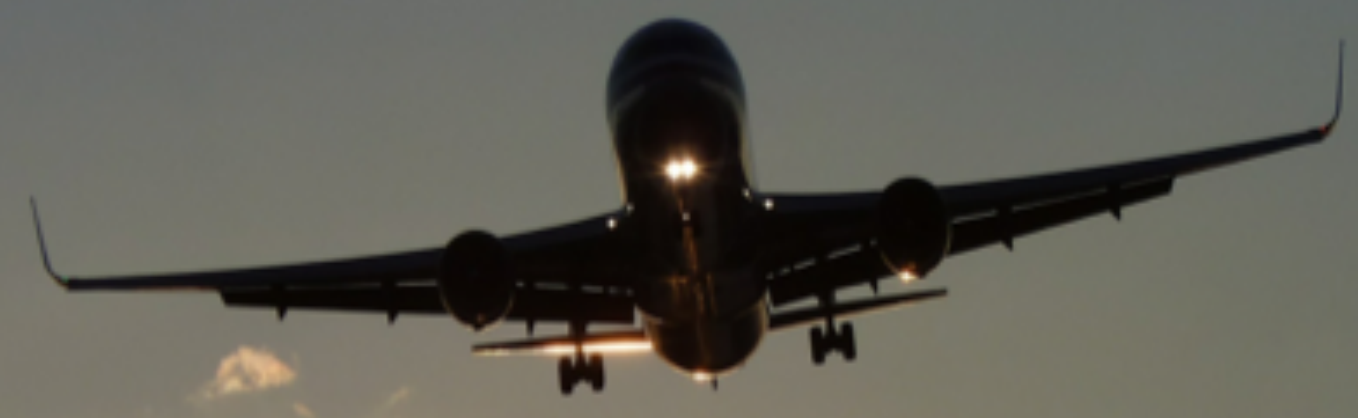

- Future Work 


\section{Summary}

- DRAW assists TMCs in issuing arrival reroutes:

- Avoid weather.

- Support arrival metering schedule.

- Improve predictability and responsiveness.

- Our laboratory evaluation demonstrated that ...

- TMC rerouted earlier when using DRAW.

- Use of DRAW reduced the number of flights with residual weather conflicts in Center airspace.

- TMCs reported their workload acceptable and DRAW generally helpful for arrival management in weather.

- TMCs somewhat agreed that DRAW would help sustaining arrival metering.

- DRAW did not increase controller workload. 
- Additional studies are planned to...

- Improve arrival metering support in weather

- Evaluate DRAW in different airspace

- Refine DRAW concepts

- DRAW simulation demonstration in the FAA's future Time-Based Flow Management (TBFM) environment has been in work. 


\section{Thank you. \\ Questions?}

Credits for the visuals used in this presentation:

- Slide template by SlidesCarnival, CC BY 4.0

- Lab photos by NASA/Dominic Hart (p. 16) \& Easter Wang (pp. 17-20)

- Sky/douds/airplane photos by Skeeze (p. 3).Koon Boh Goh (p. 7),

Rudv and Peter Skitterians (p. 12), Jonnv Lindner (p. 21),

Albert laime Casanova (p. 26), \& Michał (p. 29), downloaded from Pixabay, Cco 